\title{
Colossal Magnetoresistance in Perovskite-like La-Ca-Mn-O
}

\author{
S. Jin
}

AT\&T Bell Laboratories, Murray Hill, NJ 07974

\begin{abstract}
A colossal magnetoresistance effect with more than four orders of magnitude change in resistivity $\left(\Delta \mathrm{R} / \mathrm{R}_{\mathrm{H}}=10^{6} \%\right.$ at $\left.110 \mathrm{~K}, \mathrm{H}=6 \mathrm{~T}\right)$ has been obtained in epitaxially grown La-Ca-Mn-O thin films. The effect is negative and isotropic with respect to the field orientations. The magnetoresistance is strongly temperature-dependent, and exhibits a sharp peak that can be shifted to near room temperature by adjusting processing parameters. The very large MR effect occurs only when the film thickness is less than about 2000 A. Near-room-temperature $\Delta R / R_{H}$ values of $-1,300 \%$ at $260 \mathrm{~K}$ and $-400 \%$ at $280 \mathrm{~K}$ have been observed. The orders of magnitude change in electrical resistivity could be useful for various magnetic and electric device applications.
\end{abstract}

Keywords: magnetoresistance, thin film, epitaxy, ferromagnetic

\section{Introduction}

Colossal magnetoresistance (CMR) with the field-dependent change in electrical resistivity by many orders of magnitude has recently been discovered in $\mathrm{La}-\mathrm{Ca}-\mathrm{Mn}-\mathrm{O}$ type epitaxial thin films $s^{(1)(2)}$ as well as in Pr-Sr-Ca-Mn-O type bulk materials. ${ }^{(3)}$ Potential applications of the CMR materials in new types of devices have been discussed in recent articles ${ }^{(4)}$ Perovskite-like oxide of lanthanum manganite $\left(\mathrm{LaMnO}_{3}\right)$ exhibits both strong ferromagnetism and metallic conductivity when La ions ( $3+$ valence) are partially substituted with $2+$ valence ions such as $\mathrm{Ca}, \mathrm{Ba}, \mathrm{Sr}, \mathrm{Pb}$ and $\mathrm{Cd}$. This results in a $\mathrm{Mn}^{3+} / \mathrm{Mn}^{4+}$ mixed valence state creating mobile charge carriers and canting of $\mathrm{Mn}$ spins. ${ }^{(5)-(10)}$ The unit cell of a perovskite $\mathrm{ABO}_{3}$ is shown in Fig. 1, where $\mathrm{A}$ at the corners represents. a large ion such as $\mathrm{La}^{3+}, \mathrm{Nd}^{3+}, \mathrm{Pr}^{3+}, \mathrm{Ca}^{2+}, \mathrm{Sr}^{2+}$, $\mathrm{Ba}^{2+}, \mathrm{Pb}^{2+}, \mathrm{B}$ at the center of the cube stands for a small ion such as $\mathrm{Mn}^{3+}, \mathrm{Mn}^{4+}, \mathrm{Cr}^{3+}, \mathrm{Fe}^{3+}, \mathrm{Ti}^{4+}$, and $\mathrm{O}$ at the center of the faces represents $\mathrm{O}^{2-}$.
The magnetoresistance (MR) behavior of Lamanganites thin films has been reported for $\mathrm{La}-\mathrm{Ba}-$ $\mathrm{Mn}-\mathrm{O}^{(11)}$ (with the MR ratio of $\sim 150 \%$ at room temperature) and $\mathrm{La}-\mathrm{Ca}-\mathrm{Mn}-\mathrm{O}^{12}$ (MR ratio of $\sim 110 \%$ at $220 \mathrm{~K}$ and near zero at room temperature). The $\mathrm{MR}$ ratio is defined here as $\Delta R / R_{H}=\left(R_{H}-R_{0}\right) / R_{H}$ where $R_{0}$ is the zero field resistance and $R_{H}$ is the resistivity in the applied magnetic field, e.g., $\mathrm{H}=6 \mathrm{~T}$. These oxide films exhibited relatively small magnetoresistance comparable to the higher end of the values for the so-called "giant-magnetoresistance (GMR)" type materials of metallic multilayer or heterogeneous films with the MR values typically in the range of $5-150 \%$.

In this paper we report the electrical, magnetic and magnetoresistance behavior in epitaxial $\mathrm{La}-\mathrm{Ca}$ $\mathrm{Mn}-\mathrm{O}$ films with extraordinary MR values in excess of $10^{6} \%$, more than four orders of magnitude greater than were previously reported for the GMR-type or La-manganite films.

\section{Experimental}

La-Mn-Ca-O films, $100-5000 \AA$ thick, were deposited on (100) $\mathrm{LaAlO}_{3}$ substrates by pulsed 

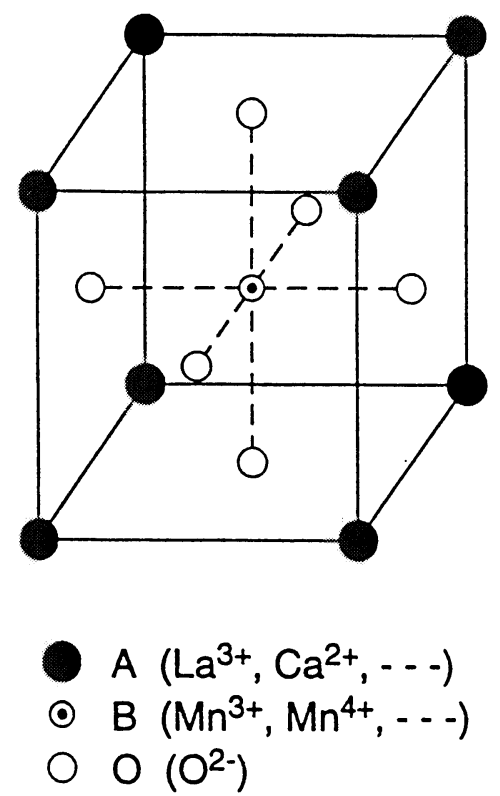

Fig. 1 Unit cell structure of manganite type perovskite.

laser deposition (PLD). The substrate temperature was about $700^{\circ} \mathrm{C}$, and the oxygen partial pressure in the chamber was maintained at 100-300 milliTorr. The nominal target composition was $\mathrm{La}_{0.67} \mathrm{Ca}_{0.33} \mathrm{MnO}_{\mathrm{x}}$. The chemical composition of the deposited film was found to be similar to that of the bulk target used for the deposition by scanning electron microanalysis and Rutherford backscattering analysis. X-ray diffraction and rocking angle analysis as well as transmission electron microscopy indicate that the films have the perovskite-type cubic structure with a lattice parameter of $\mathrm{a}=3.89 \AA$ (or $\mathrm{a}=7.78 \AA$ from the crystallographic point of view) and grow epitaxially on the $\mathrm{LaAlO}_{3}$ substrate $(\mathrm{a}=3.79 \AA)$.

The electrical resistance and magnetoresistance of the films were measured as a function of temperature and magnetic field by four point technique (using a constant current) in a superconducting magnet with the maximum applied field of $\mathrm{H}=6$ Tesla. For most of the films, the field direction was parallel to the current direction. Some of the measurements were carried out with the field applied perpendicular to the current direction in the film (either by in-plane field or perpendicular field). The MR behavior in the La$\mathrm{Ca}-\mathrm{Mn}-\mathrm{O}$ films is almost always negative and essentially isotropic with respect to the field direction if the demagnetizing factor is taken into consideration. The $\mathrm{M}-\mathrm{H}$ loops were obtained by using a vibrating sample magnetometer with the maximum field of $\mathrm{H}=1 \mathrm{~T}$. The transmission electron microscopy (TEM) was carried out by using JEOL 4000 Microscope operated at $400 \mathrm{kV}$. The samples were thinned by ion milling.

\section{Results and Discussion}

The La-Ca-Mn-O film $(-1000 \AA$ thick $)$ in asdeposited condition exhibited a MR ratio of $39,000 \%$ (at $100 \mathrm{~K}, \mathrm{H}=6 \mathrm{~T}$ ) which is substantially higher than the values of $-500 \%$ that we reported previously. Subsequent heat treatment at $850^{\circ} \mathrm{C} / 1 \mathrm{~h}$ in a $3 \mathrm{~atm}$ oxygen atmosphere dramatically improves the MR ratio to $1.1 \times 10^{6} \%$ (at $110 \mathrm{~K}$, $\mathrm{H}=6 \mathrm{~T}$ ) which is the highest value ever reported for the La-Ca-Mn-O film system. If expressed in $\Delta R / R_{0}$ (instead of $\Delta R / R_{H}$ ), the $M R$ ratios are $99.744 \%$ and $99.991 \%$ for the as-deposited and the $850^{\circ} \mathrm{C} / 1 \mathrm{~h}$ heat treated films, respectively].

In Fig. 2, the resistivity versus field curve for the heat treated La-Ca-Mn-O film at $110 \mathrm{~K}$ is shown. The figure shows that the major part of the resistivity drop occurs at $\mathrm{H}<2 \mathrm{~T}$. The zero-field resistivity of $\rho=50.8 \Omega \mathrm{cm}$ (resistance $R=4.13$ $\mathrm{M} \Omega)$ is reduced to $\rho=4.61 \mathrm{~m} \Omega \mathrm{cm}(\mathrm{R}=375 \Omega)$ when the in-plane applied field (parallel to the direction of the applied current) is increased to $6 \mathrm{~T}$.

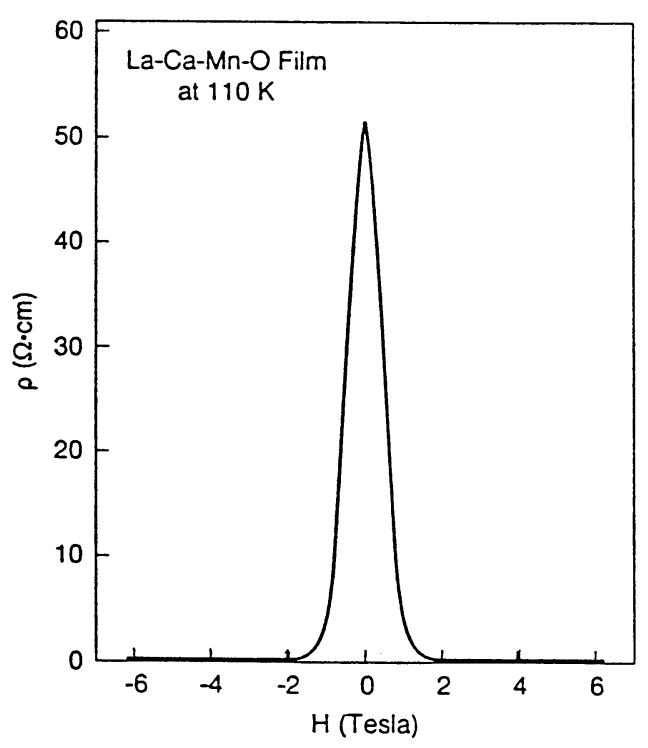

Fig. 2 Resistivity vs field curve for the La-Ca$\mathrm{Mn}-\mathrm{O}$ film at $110 \mathrm{~K}$. 
The exact reason why these samples, both the asdeposited and the heat treated films, exhibit higher MR ratios than our previous samples ${ }^{1}$ is not clearly understood at the moment. The general trend that we observed, however, is that denser and more uniform targets (e.g., sintered at higher temperatures and longer times) produce better quality films with improved epitaxy, less particulate incorporation, better chemical homogeneity and thickness uniformity, thus exhibiting higher MR values.

Interestingly, the magnetoresistance in the La$\mathrm{Ca}-\mathrm{Mn}-\mathrm{O}$ films exhibits a strong dependence on film thickness as shown in Fig. 3. The curve in Fig. 3 was constructed by using the highest attainable MR value (at 110-230 K, H =6-T) for each film thickness after heat treatments under various conditions $\left(750-950^{\circ} \mathrm{C}\right.$ for $\left.1-12 \mathrm{~h}\right)$ in an oxygen atmosphere $(3 \mathrm{~atm})$. The curve shows a maximum at a film thickness of $-1000 \AA$, with the highest MR ratio of 1.1 million \%. The MR ratios for the films on either side of the peak in Fig. 3 are drastically lower. The values for the thicker films are $-1700 \%$ for $2000 \AA$ and $-1400 \%$ for the $5000 \AA$ thickness. The films substantially thinner than $\sim 1000 \AA$ also exhibit decreased MR ratios, e.g., $-21,000 \%$ for $500 \AA$ thickness and $2200 \%$ for $100 \AA$ thickness.

It is hypothesized that the observed thickness dependence of MR is related to the change in the lattice strain induced by the change in film

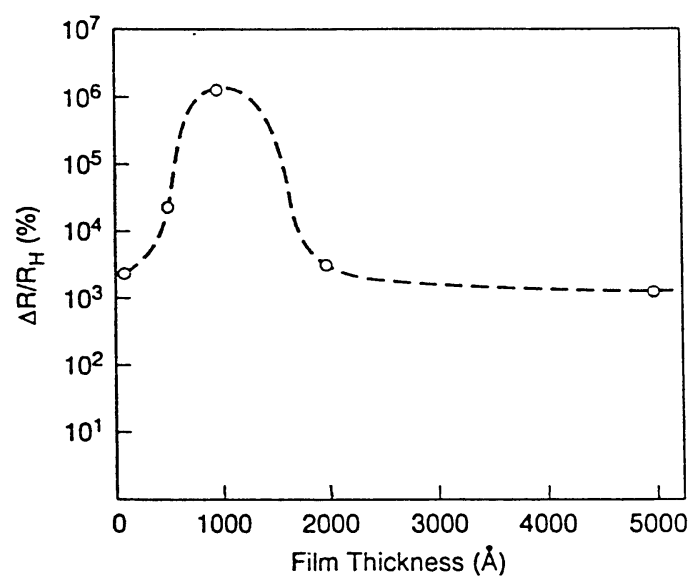

Fig. 3 Thickness dependent magnetoresistance in the La-Ca-Mn-O film. thickness. In our previous report on the MR behavior of $\mathrm{La}_{0.67} \mathrm{Ca}_{0.33} \mathrm{MnO}_{\mathrm{x}}$ thin films, ${ }^{1}$ it was pointed out that the very large MR values $(>100,000 \%)$ were obtainable, not in a polycrystalline form, but only in epitaxially grown films on single crystalline $\mathrm{LaAlO}_{3}$ with a lattice parameter of $\sim 3.791 \AA$, smaller than that of the bulk La-Ca-Mn-O materials $(\mathrm{a}-3.867 \AA)$. When deposited on polycrystalline yttria-stabilized zirconia substrates or lattice-mismatched single crystalline substrates such as $\mathrm{Si}$ or $\mathrm{MgO}$, the MR ratio of the film was very low, typically below $-500 \%$. Thus it is quite possible that the very large magnetoresistance observed is directly related to the straining of the lattice, i.e., compressively by the epitaxy imposed by the substrate with the smaller lattice parameter.

It is expected that the degree of epitaxial straining of the La-Ca-Mn-O film lattice by the $\mathrm{LaAlO}_{3}$ substrate will decrease as the film becomes thicker. The thicker films would then consist of strained, high-resistivity, high-MR region near the substrate and less strained low-resistivity, low-MR region away from the substrate. The low-resistivity region dominates the MR measurement as the applied current preferentially flows through it thus making the high-resistivity, high-MR region undetectable. The data in Fig. 3 and the aforementioned argument further support. our hypothesis that the MR behavior in the $\mathrm{La}$ manganites is closely related to optimization of the perovskite lattice parameter, which would not be surprising in that the change in lattice parameter would affect the interatomic distance and bond angle, thus influencing the magnetic or electronic exchange interactions between two magnetic cations separated by an anion.

Shown in Fig. 4 are the temperature dependence characteristics of $\rho, \Delta R / R_{H}$ and $M$ of a La-Ca$\mathrm{Mn}-\mathrm{O}$ film which was heat treated at $900^{\circ} \mathrm{C}$ and exhibits $-120,000 \%$ MR effect. The temperaturedependent behavior of the $850^{\circ} \mathrm{C}$ treated sample is similar except for higher MR values and a higher peak MR temperature of $\sim 140 \mathrm{~K}$. As is evident in the figure, the $\rho$ vs $T$ curve exhibits a relatively sharp cusp at $-95 \mathrm{~K}$ with the film showing semiconductor behavior (i.e., a negative $d \rho / d T$ ) above and metallic behavior (a positive $d \rho / d T$ ) below this temperature. The temperature at which the magnetoresistance $\left(\Delta R / R_{H}\right)$ of the film is maximum is almost invariably located in the metallic-behavior region on the left (low 


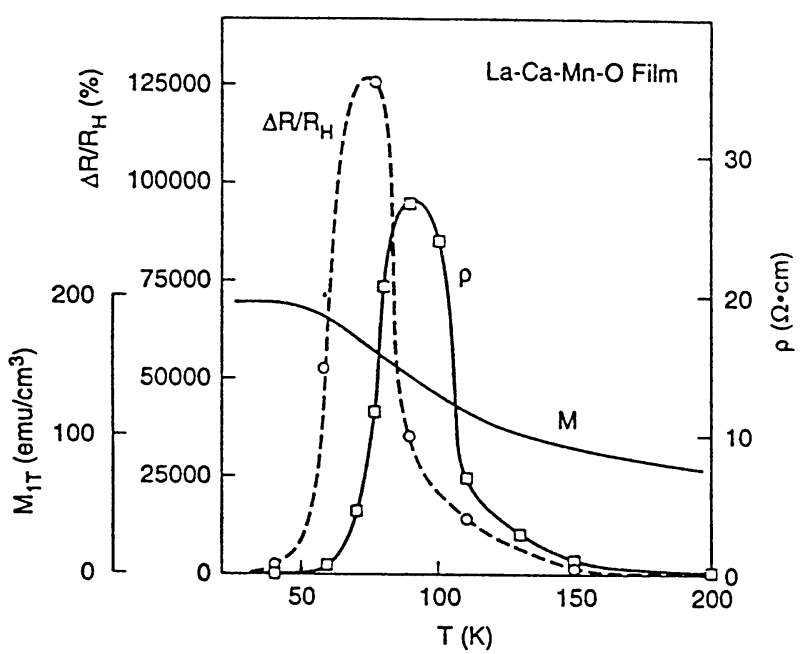

Fig. $4 \rho, \Delta R / R_{H}$ and $M$ vs $T$ curves for the epitaxial La-Ca-Mn-O film.

temperature) side of the resistivity peak. It is interesting to note that the magnetoresistance peak occurs at the temperature where the resistivity is roughly one-half of the peak resistivity or near the inflection point of the curve. The mechanism(s) responsible for the very large magnetoresistance observed in the La-Ca-Mn-O film appear(s) to be related to the semiconductor-to-metal transition, however, the exact mechanism(s) are not clearly understood at the moment.

Also shown in Fig. 4 is the magnetization $M$ (at $\mathrm{H}=1 \mathrm{~T}$ ) vs temperature curve for the $\mathrm{La}-\mathrm{Ca}-\mathrm{Mn}-\mathrm{O}$ film. It is evident that the film is strongly ferromagnetic with $\mathrm{M} \sim 200 \mathrm{emu} / \mathrm{cm}^{3}$ at $50 \mathrm{~K}$ and $\mathrm{M} \sim 100 \mathrm{emu} / \mathrm{cm}^{3}$ at $150 \mathrm{~K}$. The $\mathrm{M}-\mathrm{H}$ loops measured below the magnetic transition (Curie temp.) exhibit magnetic hysteresis with coercivity $\left(\mathrm{H}_{\mathrm{c}}\right)$ of about 30-50 Oe.

Our recent data on epitaxial films of $\mathrm{La}_{0.60} \mathrm{Y}_{0.07} \mathrm{Ca}_{0.33} \mathrm{MnO}_{\mathrm{x}}$ give very large $\mathrm{MR}$ values ${ }^{(13)}\left(10^{7}-10^{8} \%\right)$, much higher than those for the present La-Ca-Mn-O films. The Y-doped material, because of the smaller ionic diameter of $Y$, has a lattice parameter slightly smaller than that for the undoped $\mathrm{La}-\mathrm{Ca}-\mathrm{Mn}-\mathrm{O}$, but whether this is the main cause for the improved colossal magnetoresistance is unknown.

The substantially higher electrical resistivity in the La-Ca-Mn-O films, typically $2-4$ orders of magnitude higher than the metallic magnetoresistive materials such as the $80 \mathrm{Ni}-20 \mathrm{Fe}$ permalloy, could be an advantage for field sensing as the sensor voltage output $(\Delta \mathrm{V} / \Delta \mathrm{H}$ or $\Delta \mathrm{R} / \Delta \mathrm{H})$ is that much larger. The $R$ vs $H$ curve for a $\mathrm{La}_{0.55} \mathrm{Ca}_{0.25} \mathrm{Sr}_{0.08} \mathrm{MnO}_{\mathrm{x}}$ film at room temperature $(\rho-1.4 \mathrm{~m} \Omega \cdot \mathrm{cm})$ is approximately linear with an MR ratio of $4.6 \%$ at $\mathrm{H} \sim 1000$ Oe (equivalent to about $0.046 \% \mathrm{MR}$ for a lower field of $\sim 10 \mathrm{Oe}$ (this is about the level of field strength of interest for magnetic recording read head)). However, as the electrical resistance of this oxide film is about two orders of magnitude higher than that of the permalloy (with the MR ratio on the order of $3 \%$ for $\mathrm{H}=10 \mathrm{Oe}$ ), the field sensing output voltages turn out to be comparable for the two materials at the same level of sensor current. For a constant current of $1-10 \mathrm{~mA}$, the $\Delta \mathrm{V}$ voltage signal from this unoptimized film for $\mathrm{H}=10$ Oe would be 0.13-1.3 $\mathrm{mV}$, comparable to the $\Delta \mathrm{V}$ value from the permalloy film with the same dimension.

The magnetoresistive properties of the Lamanganite films can be used for a number of other applications. An example is a magnetoresistive microphone design such as schematically illustrated in Fig. 5. A small, La-Ca-Sr-Mn-O type MR sensor, about $2 \times 4 \mathrm{~mm}$ size was mounted on a plastic diaphragm. As sound waves with varying intensity hit the diaphragm and make it vibrate, moving the MR sensor in relation to the magnet that supplies a gradient field. The resulting change in the magnetic field intensity on the sensor causes change in resistivity, which in turn changes the output voltage $\Delta \mathrm{V}$. The sensor signal, many

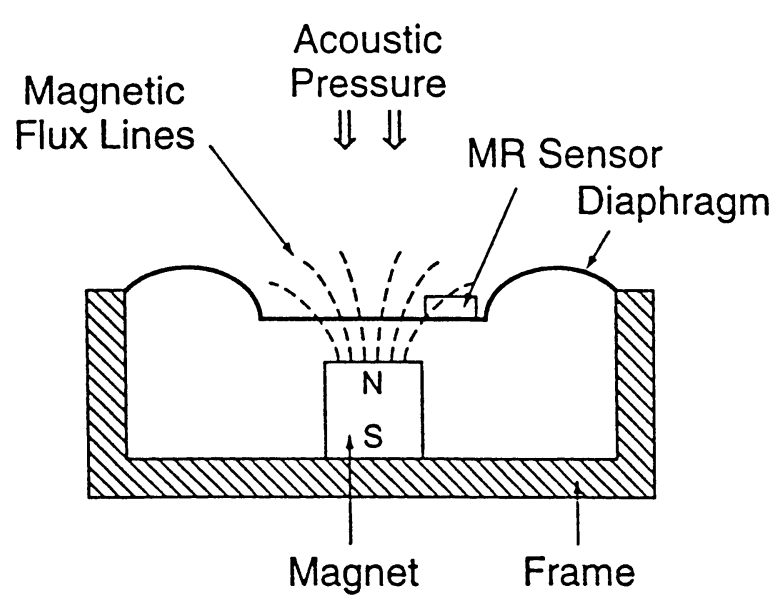

Fig. 5 A magnetoresistive microphone design. 
millivolts even in the unamplified condition, decreases with the distance from the sound source as is expected.

The MR behavior of the La-Ca-Mn-O film depends not only on the specifics of post heat treatment but also on a number of other composition and processing details including various film deposition conditions. For example, a higher oxygen partial pressure $\left(\mathrm{p}_{\mathrm{O}_{2}}\right)$ during deposition tends to produce films with higher temperature of peak resistance. The magnetization especially near room temperature also increases noticeably with $\mathrm{P}_{\mathrm{O}_{2}}$. A film deposited at $\mathrm{PO}_{2} \sim 300$ milliTorr gives near-room temperature magnetoresistance values of $\Delta R / R_{H}-1300 \%$ at $260 \mathrm{~K}$ and $-470 \%$ at $280 \mathrm{~K}$.

\section{Summary}

Colossal magnetoresistance in excess of $10^{6} \%$ has been obtained in epitaxially-grown, single crystalline $\mathrm{La}-\mathrm{Ca}-\mathrm{Mn}-\mathrm{O}$ films. These values are orders of magnitude higher than those previously reported for the GMR multilayer films and Lamanganite films. The magnetoresistance behavior appears to be related to the semiconductor-to-metal transition. The fact that the electrical resistivity of the material can be manipulated by applied field to a value orders of magnitude different could be exploited for various technical applications.

\section{REFERENCES}

1. S. Jin, T. H. Tiefel, M. McCormack, R. A. Fastnacht, R. Ramesh, and L. H. Chen, Science 264, 413 (1994).

2. S. Jin, T. H. Tiefel, M. McCormack, H. M. O'Bryan, L. H. Chen and R. Ramesh, Appl. Phys. Lett. 67, 557 (1995).

3. B. Raveau, A. Maignan, and V. Caignaert, J. Sol. State Chem. 117, 426 (1995).

4. G. Prinz and K. Hathaway, Physics Today, April 1995, p. 24.

5. G. H. Jonker and J. H. van Santen, Physica 16, 337 (1950).

6. E. O. Wollan and W. C. Koehler, Phys. Rev. 100, 545 (1955).

7. P. G. deGennes, Phys. Rev. 118, 141 (1960).

8. J. B. Goodenough, Phys. Rev. 100, 564 (1955).

9. B. C. Tofield and W. R. Scott, J. Solid State Chem. 10, 183 (1974).

10. C. W. Searle and S. T. Wang, Canadian J. Phys. 48, 2023 (1970).

11. R. von Hemlolt, J. Wecker, B. Holzapfel, L. Schultz, K. Samwer, Phys. Rev. Lett. 71, 2331 (1993).

12. K. Chahara, T. Ohno, M. Kasai, and Y. Kozono, Appl. Phys. Lett. 63, 1990.

13. L. H. Chen, S. Jin, T. T. M. Palstra (unpublished). 